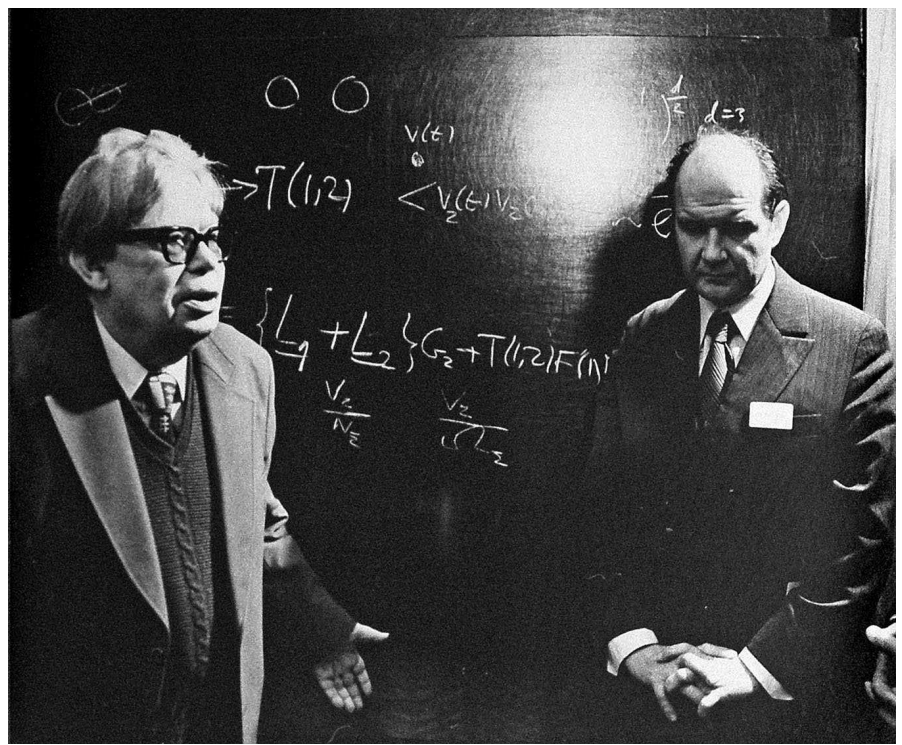

\title{
Mykola Bogolyubov and Lviv School of Statistical Physics
}

Mykola Bogolyubov was undoubtedly a man of genius. He has a unique impact on his surroundings through the strength of his logical reasonings, his generosity and the tranquility that emanated from him. How does it come about with a man? Perhaps this lies in the individual's constitution, his or her genetic code as well as in the external circumstances. But even the influence of external circumstances essentially depends on the extent of their apprehension. For the youthful Bogolyubov these essential circumstances were his family. He perceived their influence by all the fibers of his being. Everything like sound, sight, touch and movement was familiar to him, indispensable, warm, tasty, logical, pleasant and just, because it was his. His parents were persons of talent. His father was a philosopher, theologist, historian and literary scholar who knew many European languages. His mother was a musician, a gifted pianist. Gospel truth of Christian moral precepts was introduced by the Bogolyubov family into the consciousness of the youthful Mykola. And, of course, a beneficial atmosphere of the secondary school in Velyka Krucha, an enchanting village in Poltava region, where he was lucky enough to live among kindly souls and have wise and skilled teachers. He found himself in a dense atmosphere of colorful Ukrainian language, in the atmosphere of Taras Shevchenko's poetry and splendid folk singing. Mykola didn't have the slightest reason to resist this beneficial influence. He gratefully accepted it and made himself the academician Bogolyubov whom we know to be our great teacher.

Bogolyubov's impact on the Lviv school of theoretical physics was induced by his well-known book "Problems of dynamic theory in statistical physics" that was published in 1946. It was a young scientist Abba Glauberman, a postgraduate from Leningrad (now St. Peterburg), who in 1948 turned our attention upon this book here in Lviv. He started lecturing a special physics course in perfect Ukrainian language. This young, dark-haired Jewish man was lucky to survive from the Nazis here in Ukraine. Glauberman supervised my diploma paper and my postgraduate work which dealt with Bogolyubov's expansions for the distribution function of a system of charged particles. In my first postgraduate year I managed to publish a paper in the most prestigious physical journal in the USSR.

Having hand-written my candidate dissertation in a nice big notebook, I brought it to Moscow University to find the seminar conducted by Bogolyubov. After the seminar I came up to Bogolyubov and asked him if he could possibly look through my dissertation. Bogolyubov agreed and 
told me to come the next day. The next day he told me that everything was all right and that I could defend it. He also agreed to be my official opponent for the defence.

This is how Bogolyubov directly entered my life and the life of theoretical physicists in Lviv. Another great mathematician and physicist belonging to Bogolyubov's school entered our scientific life in 1954 after defending his candidate dissertation. It was Ostap Parasyuk. Within the quantum field theory there is a well-know Bogolyubov-Parasyuk theorem. Parasyuk suggested me to get acquainted with a small article of another well-known Bogolyubov's disciple, professor Zubarev. In fact, this work launched the development of mathematical apparatus of Lviv school of statistical physics. We managed to obtain an explicit expression for the Jacobian, which is a key function that describes the change of the scale at transition in integrals from a system of fixed number of coordinates to the wave function. The Jacobian had an initial form of infinite product of generalized functions. It was really a hard problem to be solved which took me about three years of continuous hourly labour to cope with. I published it and again received Bogolyubov's acknowledgement. From then on all my pupils' dissertations got Bogolyubov's approval. There took shape unceasing relations of Lviv with the Steklov Mathematics Institute in Moscow and soon with the scientific center in Dubna.

Having become director of the Institute for Theoretical Physics in Kyiv, Bogolyubov recommended to set up a branch of the institute in Lviv. This idea was substantially furthered by the corresponding member of the Academy of Sciences V.P. Shelest. At first the Lviv department consisted of only two persons - me and a laboratory assistant. This was late in 1960-ies. Enrolment of personnel for the department was quite natural. Theoretical physics is a hard science. It can be mastered only by robust and intelligent. That is how, due to a very close interaction with Bogolyubov and with his wonderful team in Dubna, our Lviv-Dubna school of statistical physics developed.

Let me make a brief presentation of the school. Statistical theory of liquids and solutions, soft matter physics - these earliest fields of research are now continued under the supervision of my former postgraduate, now a corresponding member of the National Academy of Sciences of Ukraine Myroslav Holovko. His team including doctors of sciences Orest Pizio, Yuri Kalyuzhnyi, Andrij Kovalenko, Andrij Trokhymchuk and others managed to develop an original method of computing thermodynamic and structural functions of liquids and soft matter.

Another wide field of research is theory of phase transitions. The method of collective variables developed by us in late 1950-ies has shown all its effectiveness. As a result, the phase transitions are described in magnets, ferroelectrics, in the liquid-gas critical point, in binary mixtures of particles, etc. There has grown a wonderful group of now well-known and independent theoretical physicists, doctors of sciences: Ivan Vakarchuk, Yurij Rudavskii, Zenovyi Gurskii, Mykhailo Kozlovskii, Ihor Stasyuk, Mykola Korynevskii, Yurij Holovatch, Ihor Mryglod, Yurij Kozitskii, Ihor Idzyk, Oksana Patsahan, Ihor Pylyuk and others.

In early 1960-ies here in Lviv there was developed a method of displacements and collective variables. It is well known that because of the quantum nature of elementary particles there operates the principle of indeterminacy. The consequence of the principle is the replacement of the notion of point particle by the notion of wave packet. The lower is the average velocity of the particles, the larger is the radius of the wave packet. At low temperatures, when the average kinetic energy of particles tends to zero, the interactions between the quantum packets become determinative. Here perfect research has been done for helium at low temperatures by professor Ivan Vakarchuk and his followers. Remarkable progress has been achieved for Fermi particles by professor Markian Vavrukh, and for quasirelativistic systems by professor Lavrentyi Blazhyevskyi.

A separate talk deserves the Bogolyubov school of unequilibrium processes. The Lviv scientific team became the follower of one of the most remarkable Bogolyubov's disciples - Dmytro Zubarev. Personal contacts of the Ph.D. student Mykhailo Tokarchuk with Dmytro Zubarev caused the formation of an ever growing team researching unequilibrium statistics here in Lviv. The team includes doctors of sciences Mykhailo Tokarchuk, Ihor Mryglod, Taras Bryk, Ihor Omelyan, Petro Kostrobij.

Now comes another trend in the Lviv school of statistical physics. Corresponding member of the 
National Academy of Sciences of Ukraine Ihor Stasyuk is a recognized scientist in the scientific world who has set up a school in the field of quantum theory of strongly correlated electron systems. It should be noted that the method of local operators of creation and annihilation appeared originally in Lviv. The authors of the method were professor Glauberman's young postgraduates Vadym Vladimirov and Ihor Stasyuk. Ihor Stasyuk managed to evolve the modern methods of quantum statistics based on Green functions that are constructed on Hubbard operators (this name is now commonly used for the operators introduced by Vladimirov and Stasyuk). A wide spectrum of problems for quantum crystals with hydrogen bonds is being treated in this group which includes doctors of sciences Ihor Stasyuk, Roman Levitskyi, Oleg Derzhko, Andrij Shvaika.

All these scientific trends made up the basis for the Institute for Condensed Matter Physics of the National Academy of Sciences of Ukraine, which was set up in 1990 on the base of the Lviv department of statistical physics of the Institute for Theoretical Physics. At the Institute there operates the first, among the academic institutions in Ukraine, powerful network of rapid electronic communication UARNET (director Ihor Protsykevich, professor Holovko's pupil). By topology, technological parameters and the quality of providing the services, UARNET enters the top Ukrainian providers according to the rating of International associations CAIDA.

Since 1993 the Institute for Condensed Matter Physics publishes a quarterly journal "Condensed Matter Physics" (in English). Recognized by the European Physics Society, since 2005 it is included in the Thomson Routers Master Journal List and became the first scientific journal in the independent Ukraine that received the ISI impact factor in 2007. Its brilliant editorial board has lead the journal to the highest world standards.

Modern theoretical physics cannot proceed without the aid of computer technologies. Having earned some amount of money after solving a number of problematic tasks for the Chornobyl "Shelter"-site in 2001, Institute for Condensed Matter Physics was able to create the first in the National Academy of Sciences of Ukraine powerful cluster with productivity of 12.5 Gflops, equipped with 32 single-kernel processors. In 2009 the peak productivity of the cluster already reaches 928 Gflops and there is an in-built storing server with the general memory capacity of 3 Tbyte. A young doctor of sciences Taras Bryk, having gone through a wonderful experience of working with powerful computers in scientific centers in Europe and the USA, is at the head of the development of computer modelling in the Institute.

And, finally, the issue of delegating the powers within the Institute. The fact is that each of the senior generation of doctors used to hold the post of the deputy chief of the Lviv department of statistical physics for at least 1 or 2 years. It was in early 1980-ies that I singled out Ihor Mryglod from the group of fourth year students of the physics faculty at Lviv university. I was leading this young personality. He is erudite, calm, just and quite steadfast. Now he is a corresponding member of the National Academy of Sciences of Ukraine and director of the Institute. Deputy directors of the Institute are Taras Bryk and Mykola Patsahan, scientific secretary is Roman Melnyk. The Institute is bound to develop further.

Having reviewed the team of the Institute for Condensed Matter Physics I haven't mentioned its new works and trends. Here the Institute regularly co-operates with scientists from France, Russia, USA, Austria, Germany, Japan, Canada, Poland and other countries. There is a journal and a high international citation index of their papers. We can state with satisfaction that Mykola Bogolyubov would be really glad and proud of having in 1969 set up in Lviv the department of Statistical Theory of Condensed States of the Kyiv Institute of Theoretical Physics. We have surely lived up to his expectations.

Ihor Yukhnovskii ${ }^{1}$

\footnotetext{
${ }^{1}$ Prof. I. Yukhnovskii was a founder of the Institute for Condensed Matter Physics of the National Academy of Sciences of Ukraine and director of the Institute from 1990 till 2006 (Ed.).
} 
